\title{
Guidelines
}

\section{Swiss Recommendations for the Follow-Up and Treatment of Pulmonary Long COVID}

\author{
Manuela Funke-Chambour ${ }^{a}$ b Pierre-Olivier Bridevaux ${ }^{c, d}$ Christian F. Clarenbach $^{e}$ \\ Paola M. Soccal ${ }^{d, f}$ Laurent P. Nicod ${ }^{g, h} \quad$ Christophe von Garnier $^{\text {h }}$ on behalf of the Swiss \\ COVID Lung Study Group and the Swiss Society of Pulmonology

\begin{abstract}
a Department for Pulmonary Medicine, Inselspital, Bern University Hospital, University of Bern, Bern, Switzerland; ${ }^{b}$ Department for Pulmonary Medicine, BioMedical Research, University of Bern, Bern, Switzerland; ' Service de Pneumologie, Hôpital du Valais, Sion, Switzerland; ' ${ }^{\mathrm{d}}$ Geneva Medical School, University of Geneva, Geneva, Switzerland; ${ }^{e}$ Department of Pulmonology, University Hospital Zurich, Zurich, Switzerland; ${ }^{\mathrm{f} D i v i s i o n}$ of Pulmonary Medicine, Geneva University Hospitals, Geneva, Switzerland; 9 Pulmonary Medicine, Clinique Cecil du groupe Hirslanden, Lausanne, Switzerland; ' Division of Pulmonary Medicine, Lausanne University Hospital (CHUV), University of Lausanne, Lausanne, Switzerland
\end{abstract}

\section{Keywords}

COVID sequelae · Post-COVID clinics · Long COVID · Postacute COVID · Long COVID recommendations · COVID-19 . SARS-CoV-2 recommendations · Interim guidance

\footnotetext{
Abstract

Introduction: Emerging evidence suggests that long-term pulmonary symptoms and functional impairment occurs in a proportion of individuals following SARS-CoV-2 infection. Although the proportion of affected patients remains to be determined, physicians are increasingly being confronted with patients reporting respiratory symptoms and impairment beyond the acute phase of COVID-19. In face of limited evidence, the Swiss Society for Pulmonology established a working group to address this area of unmet need and formulated diagnostic and treatment recommendations for the care of patients with pulmonary long COVID (LC). Method: The Swiss COVID Lung Study group and Swiss Society for Pulmonology (SSP) formulated 13 questions addressing the
}

diagnosis and treatment of pulmonary LC. A survey within the SSP special interest groups involved in care of LC patients was conducted in Switzerland. A CORE process/Delphi-like process was used to formulate recommendations. Forty experienced pulmonologists replied to the first survey and 22 completed the second follow-up survey. Agreement of $\geq 70 \%$ consensus led to formulation of a recommendation. Results: The participants in the survey reached consensus and formulated a strong recommendation for regarding the following points. Patients hospitalized for COVID-19 should have a pulmonary assessment including pulmonary function tests. Symptomatic subjects affected by COVID-19, including those with mild disease, should benefit from a pulmonary follow-up. Persistent respiratory symptoms after COVID-19 should be investigated by a pulmonary follow-up including plethysmography, diffusion capacity measurement, and blood gases analysis. Individuals having suffered from COVID-19 and who present with persistent respiratory symptoms should be offered a rehabilitation. Additional questions were given moderate or weak recommendations
(C) 2021 The Author(s)

Published by S. Karger AG, Basel

This is an Open Access article licensed under the Creative Commons Attribution-NonCommercial-4.0 International License (CC BY-NC) (http://www.karger.com/Services/OpenAccessLicense), applicable to the online version of the article only. Usage and distribution for commercial purposes requires written permission.
Correspondence to:

Manuela Funke-Chambour, manuela.funke-chambour@insel.ch 
for. The panel did not reach sufficient consensus for pharmacological therapy (e.g., therapy specifically targeting lung fibrosis) to formulate recommendations for LC drug treatment. Conclusion: The formulated recommendations should serve as an interim guidance to facilitate diagnosis and treatment of patients with pulmonary LC. As new evidence emerges, these recommendations may need to be adapted.

(c) 2021 The Author(s)

Published by S. Karger AG, Basel

\section{Summary of Recommendations}

Question 1: Should All Patients Hospitalized for COVID-19 Have a Pulmonary Follow-Up?

- All patients hospitalized for COVID-19 should have a pulmonary follow-up. (strong recommendation for, consensus reached)

- The suggested time frame is within 3 months after infection. (suggestion, no consensus)

Question 2: Should All COVID-19 Affected Patients Including Those with Mild Disease Have a Pulmonary Follow-Up, if Symptomatic?

- All COVID-19 affected patients, including those with mild disease, should have a pulmonary follow-up, if symptomatic. (strong recommendation for, consensus reached)

- The suggested time frame is within 3 months after infection. (suggestion, no consensus)

Question 3: Should All Patients with Persistent Respiratory Symptoms after COVID-19 Have a Pulmonary Follow-Up Including Plethysmography, Diffusion Capacity Measurement, and Blood Gases Analysis?

- All patients with persistent respiratory symptoms after COVID-19 should have a pulmonary follow-up including plethysmography, diffusion capacity measurement, and blood gases analysis. (strong recommendation for, consensus reached)

Question 4: Should All Patients with Severe COVID-19 (WHO Definition) Have a Pulmonary Follow-Up Including Plethysmography and Diffusion Capacity, Blood Gases Analysis Independent of Symptom Persistence?

- For patients with severe COVID-19 (WHO definition), a pulmonary follow-up is recommended including plethysmography and diffusion capacity, blood gases analysis independently of symptom persistence. (moderate recommendation for, consensus reached when strong and weak recommendations are merged)

- Remark: Consensus was reached when strong and weak recommendations are merged, $64 \%$ gave a strong recommendation for and $36 \%$ a weak recommendation for.

Question 5: Should Exercise Testing (e.g., 6MWD or

Equivalent) be Routinely Performed after COVID-19?

- Exercise testing (e.g., $6 \mathrm{MWD}$ or equivalent) is recommended after COVID-19. (weak recommendation for, consensus reached)

Swiss Recommendations for Pulmonary

Long COVID
Question 6: Should Chest CT Scan Be Routinely Performed in Patients with Persisting Respiratory Symptoms after COVID-19?

- Chest CT scan is recommended to be routinely performed in patients with persisting respiratory symptoms after COVID-19. (moderate recommendation for, consensus reached when strong and weak recommendations are merged)

- Remark: Consensus was reached when strong and weak recommendations are merged, $68 \%$ gave a strong recommendation for and $32 \%$ a weak recommendation for.

Question 7: Should Cardio-Pulmonary Exercise Test Be Routinely Performed after COVID-19 with Persistent Symptoms?

- Cardio-pulmonary exercise test (CPET) is recommended to be routinely performed after COVID-19 with persistent symptoms. (moderate recommendation for, consensus reached when strong and weak recommendations are merged)

- Remark: Consensus was reached when strong and weak recommendations are merged, $14 \%$ gave a strong recommendation for and $68 \%$ a weak recommendation for.

Question 8: Should Patients with Persistent Symptoms after COVID-19 Have Access to Specialized Multidisciplinary PostCOVID-19 Clinics or Networks?

- Patients with persistent symptoms after COVID-19 are recommended to have access to specialized multidisciplinary PostCOVID-19 clinics or networks. (moderate recommendation for, consensus reached when strong and weak recommendations are merged)

- Remark: Consensus was reached when strong and weak recommendations are merged, $55 \%$ gave a strong recommendation for and $41 \%$ a weak recommendation for.

Question 9: Should Patients after COVID-19 Who Present with New Onset Obstructive Lung Disease Have Empiric Topic Inhaled or Systemic Steroid Treatment?

- Patients after COVID-19 who present with new obstructive lung disease are recommended to be offered empiric topic inhaled or systemic steroid treatment. (moderate recommendation for, consensus reached when strong and weak recommendations are merged)

- Remark: Consensus was reached when strong and weak recommendations are merged, $36 \%$ gave a strong recommendation for and $36 \%$ a weak recommendation for.

Question 10: Should Patients after COVID-19 Who Present with Persistent Cough Have Empiric Inhaled Topic Steroids?

- Patients after COVID-19 who present with persistent cough are recommended to have empiric inhaled topic steroids. (moderate recommendation for, consensus reached when strong and weak recommendations are merged)

- Remark: Consensus was reached when strong and weak recommendations are merged, $32 \%$ gave a strong recommendation for and $45 \%$ a weak recommendation for. 
Table 1. Modified proposal for the definition of acute and long COVID [15]

\begin{tabular}{ll}
\hline Acute COVID-19 & Signs and symptoms of COVID-19 for up to 4 weeks \\
\hline Ongoing symptomatic COVID-19 & Signs and symptoms of COVID-19 from 4 weeks up to 12 weeks \\
\hline Post-COVID-19 syndrome & $\begin{array}{l}\text { Signs and symptoms that develop during or after an infection consistent with COVID-19, } \\
\text { continue for more than 12 weeks and are not explained by an alternative diagnosis }\end{array}$ \\
\hline Long COVID & $\begin{array}{l}\text { Long COVID describes signs and symptoms that continue or develop after acute COVID-19; } \\
\text { it includes both ongoing symptomatic COVID-19 (from 4 to 12 weeks) and post-COVID-19 } \\
\text { syndrome (12 weeks or more) }\end{array}$ \\
\hline
\end{tabular}

Question 11: Should Patients after COVID-19 Who Present with Interstitial Abnormalities after Exclusion of an Active Infection Receive an Empiric Systemic Steroid Trial?

- Patients after COVID-19 who present with interstitial abnormalities after exclusion of an active infection are recommended to be evaluated to receive an empiric systemic steroid trial. (moderate recommendation for, consensus reached when strong and weak recommendations are merged)

- Remark: Consensus was reached when strong and weak recommendations are merged, $14 \%$ gave a strong recommendation for and $59 \%$ a weak recommendation for.

Question 12: Should Patients after COVID-19 Who Present with Signs of Pulmonary Fibrosis Receive Specific Antifibrotic Drugs?

- It is unclear whether patients after COVID-19 who present with signs of pulmonary fibrosis should receive specific antifibrotic drugs. (suggestion for no recommendation, no consensus)

Question 13: Should Patients after COVID-19 Who Present with Persistent Respiratory Symptoms Undergo a Rehabilitation Program?

- Patients after COVID-19 who present with persistent respiratory symptoms are recommended to undergo a rehabilitation program. (strong recommendation for, consensus reached)

\section{Introduction}

Previous Coronavirus infections, including Severe Acute Respiratory Syndrome (SARS) [1] and Middle East respiratory syndrome (MERS), [2] were associated with a high mortality, pulmonary complications including acute respiratory distress syndrome (ARDS), and long-term sequelae in survivors $[3,4]$. COVID-19 caused by SARS-CoV2, has a similar initial clinical presentation as SARS and MERS. Initial symptoms include fever, cough and dyspnea, and bilateral opacities may be observed radiologically. ARDS is also a major respiratory complication in COVID-19, particularly in elderly patients $[5,6]$. ARDS itself can result in pulmonary sequelae and long-term impairment [7].
Whether pulmonary sequelae after coronavirus infections are linked to ARDS [7] or are related to virus-specific mechanisms of disease remains to be clarified $[8,9]$.

Cumulative literature addresses persisting pulmonary and extrapulmonary symptoms after acute COVID-19 infection $[10,11]$. These symptoms and associated organ manifestations are increasingly referred to as long COVID (LC) syndrome, although a universal definition is still missing. The post-acute COVID-19 period is considered in the presence of symptoms up to 3 weeks after infection, while LC persists beyond 12 weeks after infection $[12,13]$.

The characteristics and time-points of LC manifestations vary between 3 and 12 weeks after acute infection $[12,13]$. Persistence of symptoms that last more than 2 or 3 months beyond the COVID-19 infection without alternative diagnosis have been described [14, 15]. A modified proposal for a definition according to these recent publications is shown in Table 1 . The causes for symptom persistence and organ impairment are unknown, but hypotheses range from immunological phenomena, persistent subclinical virus infection, post-ICU syndrome to diffuse thrombotic damage due to endotheliitis $[13,16]$.

Long COVID-19 treatment programs and multidisciplinary/interdisciplinary LC consultations are increasingly being set up [17]. Although calls are made and efforts initiated to formulate guidance for physicians for treating patients with persistent symptoms or LC $[18,19]$, scientific evidence to support clinically meaningful guidelines is sparse. Switzerland has been one of the first European countries affected by the ongoing pandemic of COVID-19 in spring 2020. Persistence of respiratory symptoms 4 months after the infection has been investigated within a Swiss wide national observational study for lung sequelae (Swiss COVID Lung Study: NCT04581135), showing that respiratory impairment is most pronounced in patients following severe disease [20]. Diagnostic and treatment approaches of patients after COVID-19 have not been defined yet. 


\section{Methods}

The Swiss COVID Lung Study group and SSP have conducted a survey within its special interest group (SIG) involved in care of COVID-19 patients to evaluate interim recommendations by its members for treatment of individuals with LC. Members of the SSP SIG Sleep Breathing Disorders, Prevention (tobacco/environment), Pulmonary Vascular Disease, Obstructive Lung Diseases \& Allergy, Thoracic Interventions \& Oncology, Infections, Tuberculosis, CF/non-CF Bronchiectasis, Functional Diagnostics and Sports Pulmonology, Interstitial and Rare Lung Diseases and Ventilation and $\mathrm{O}_{2}$ participated in the survey, and names of participants are listed in the Acknowledgements.

In absence of clinical evidence, the Convergence of Opinion on Recommendations and Evidence (CORE) process/Delphi-like process is an adequate tool to formulate recommendations for physicians that need to be adapted before evidence accumulates and international consensus becomes available [21]. Questions were formulated with PICO (Population, Intervention, Comparison, and Outcome) format by an expert core committee listed as authors. The questions were discussed in a video conference on January 25th, 2021 by the participants to ensure that they accurately conveyed their intent, were clearly written, and all terms have been defined. Due to the current pandemic with restricted possibilities for face-to-face exchange, subsequent discussions were conducted virtually or by email exchange.

The electronic survey was conducted by LamaPoll ${ }^{\circledR}$ sent to all members of the SSP SIGs listed above. For each question, the respondent had to choose among a strong or weak recommendation for or against the course of action or no recommendation; participants also had the opportunity to provide written comments.

A second survey was subsequently issued that was identical to the first, with the exception that numerical responses and comments from the first survey were provided.

Agreement of $\geq 70 \%$ consensus led to formulation of a recommendation. If consensus could not be reached, no recommendation was provided, but a suggestion was formulated, if possible. If a majority of the survey participants decided for a strong or weak recommendation ( $\geq 70 \%$ if combined), consensus for a recommendation for was assumed and a moderate recommendation was provided.

The committee held another videoconference to discuss the survey results on March 31st, 2021. The sum- mary and conclusions of this discussion were integrated into the manuscript.

A total of 40 pulmonologists from all parts of Switzerland responded to the first and 22 participated in the second survey. Most pulmonologist had extensive experience with acute COVID-19 patients (42\% in the first and 55\% in the second survey with $>50$ acute COVID-19 patients). More than $56 \%$ (68\% in the second survey) treated up to 50 LC patients at the time of the survey, while only $14 \%$ were in charge of more than 50 LC patients in both surveys. This discrepancy between acute and LC care may be related to the time point of the survey before the end of the second wave in February 2021. As experience with LC was more extensive in the second survey and probably physicians with specific interest in pulmonary LC replied the second round, the results of the second survey were used to define consensus for recommendations.

\section{Results of the Swiss National Survey among Pulmonologists}

An overview of the recommendations and suggestions is provided in a summarized form including individual survey results in Table 2.

\section{Question 1: Should All Patients Hospitalized for COVID-19 Have a Pulmonary Follow-Up?}

Literature Review

Most patients hospitalized for COVID-19 pneumonia have persistent symptoms after 3-4 months [22]. Fortytwo percent of these patients report dyspnea, indicating persistent pulmonary impairment after acute infection [22]. In a Norwegian study 3 months after COVID-19, symptoms persisted in half of the patients with one-quarter having reduced diffusion capacity [23]. These findings were also confirmed by an Austrian study group [24]. In a Swiss study, specifically those with ARDS showed lung functional impairment with mainly reduced diffusion capacity 4 months after acute infection [20]. After severe COVID-19, lung functional abnormalities with impaired diffusion capacity persisted until 6 months in a recent publication [25]. Pulmonary follow-up was already suggested early in the pandemic before evidence for pulmonary sequelae emerged [26]. National German S3 Recommendations for treatment of hospitalized COVID patients suggest a clinical follow-up after 8-12 weeks to detect long-term sequelae, if possible within a study or registry [27]. 
Table 2. Overview of the voting results for each question from both surveys

\begin{tabular}{|c|c|c|}
\hline Question & Survey results 1 & Survey results 2 \\
\hline $\begin{array}{l}\text { 1. Should all patients hospitalized for COVID-19 have a } \\
\text { pulmonary follow-up? } \\
\text { At what time point should respiratory tests be performed? }\end{array}$ & $\begin{array}{l}\text { Strong recommendation for } \\
\text { consensus reached }(70 \%) \\
\mathbf{3} \text { months } \\
\text { no consensus }(49 \%)\end{array}$ & $\begin{array}{l}\text { Strong recommendation for } \\
\text { consensus reached }(77 \%) \\
\mathbf{3} \text { months } \\
\text { no consensus }(62 \%)\end{array}$ \\
\hline $\begin{array}{l}\text { 2. Should all COVID-19 affected patients including those with } \\
\text { mild disease have a pulmonary follow-up, if symptomatic? } \\
\text { At what time point should respiratory tests be performed? }\end{array}$ & $\begin{array}{l}\text { Strong recommendation for } \\
\text { consensus reached }(74 \%) \\
3 \text { months } \\
\text { no consensus }(48 \%)\end{array}$ & $\begin{array}{l}\text { Strong recommendation for } \\
\text { consensus reached }(82 \%) \\
3 \text { months } \\
\text { no consensus }(62 \%)\end{array}$ \\
\hline $\begin{array}{l}\text { 3. Should all patients with persistent respiratory symptoms after } \\
\text { COVID-19 have a pulmonary follow-up including } \\
\text { plethysmography, diffusion capacity measurement, and blood } \\
\text { gases analysis? }\end{array}$ & $\begin{array}{l}\text { Strong recommendation for } \\
\text { consensus reached }(91 \%)\end{array}$ & $\begin{array}{l}\text { Strong recommendation for } \\
\text { consensus reached }(100 \%)\end{array}$ \\
\hline $\begin{array}{l}\text { 4. Should all patients with severe COVID-19 (WHO definition) } \\
\text { have a pulmonary follow-up including plethysmography and } \\
\text { diffusion capacity, blood gases analysis independently of } \\
\text { symptom persistence? }\end{array}$ & $\begin{array}{l}\text { Moderate recommendation for } \\
\text { consensus reached when merged } \\
\text { ( } 49 \% \text { strong recommendation for } 44 \% \text { weak } \\
\text { recommendation for) }\end{array}$ & $\begin{array}{l}\text { Moderate recommendation for } \\
\text { consensus reached when merged ( } 64 \% \\
\text { strong recommendation for } 36 \% \text { weak } \\
\text { recommendation for) }\end{array}$ \\
\hline $\begin{array}{l}\text { 5. Should exercise testing (e.g., } 6 \mathrm{MWD} \text { or equivalent) be } \\
\text { routinely performed after COVID-19? }\end{array}$ & $\begin{array}{l}\text { Moderate recommendation for } \\
\text { consensus reached when merged } \\
\text { ( } 26 \% \text { strong recommendation for } 51 \% \text { weak } \\
\text { recommendation for) }\end{array}$ & $\begin{array}{l}\text { Weak recommendation for } \\
\text { consensus reached } \\
\text { (0\% strong recommendation for } 77 \% \\
\text { weak recommendation for) }\end{array}$ \\
\hline $\begin{array}{l}\text { 6. Should chest CT scan be routinely performed in patients with } \\
\text { persisting respiratory symptoms after COVID-19? }\end{array}$ & $\begin{array}{l}\text { Moderate recommendation for } \\
\text { consensus reached when merged } \\
\text { ( } 40 \% \text { strong recommendation for } 42 \% \text { weak } \\
\text { recommendation for) }\end{array}$ & $\begin{array}{l}\text { Moderate recommendation for } \\
\text { consensus reached when merged } \\
\text { (68\% strong recommendation for } 32 \% \\
\text { weak recommendation for) }\end{array}$ \\
\hline $\begin{array}{l}\text { 7. Should cardio-pulmonary exercise test (CPET) be routinely } \\
\text { performed after COVID-19 with persistent symptoms? }\end{array}$ & $\begin{array}{l}\text { Weak suggestion for } \\
\text { no consensus } \\
\text { ( } 23 \% \text { strong recommendation for } 42 \% \text { weak } \\
\text { recommendation for) }\end{array}$ & $\begin{array}{l}\text { Moderate recommendation for } \\
\text { consensus reached when merged } \\
\text { ( } 14 \% \text { strong recommendation for } 68 \% \\
\text { weak recommendation for) }\end{array}$ \\
\hline $\begin{array}{l}\text { 8. Should patients with persistent symptoms after COVID have } \\
\text { access to specialized multidisciplinary Post COVID-19 clinics or } \\
\text { networks? }\end{array}$ & $\begin{array}{l}\text { Moderate recommendation for } \\
\text { consensus reached when merged } \\
\text { ( } 65 \% \text { strong recommendation for } 8 \% \text { weak } \\
\text { recommendation for) }\end{array}$ & $\begin{array}{l}\text { Moderate recommendation for } \\
\text { consensus reached when merged } \\
\text { (55\% strong recommendation for } 41 \% \\
\text { weak recommendation for) }\end{array}$ \\
\hline $\begin{array}{l}\text { 9. Should patients after COVID-19 who present with new } \\
\text { obstructive lung disease have empiric topic inhaled or systemic } \\
\text { steroid treatment? }\end{array}$ & $\begin{array}{l}\text { Moderate recommendation for } \\
\text { consensus reached when merged } \\
\text { ( } 33 \% \text { strong recommendation for } 37 \% \text { weak } \\
\text { recommendation for) }\end{array}$ & $\begin{array}{l}\text { Moderate recommendation for } \\
\text { consensus reached when merged } \\
\text { ( } 36 \% \text { strong recommendation for } 36 \% \\
\text { weak recommendation for) }\end{array}$ \\
\hline $\begin{array}{l}\text { 10. Should patients after COVID- } 19 \text { who present with persistent } \\
\text { cough have empiric inhaled topic steroids? }\end{array}$ & $\begin{array}{l}\text { Weak suggestion for } \\
\text { no consensus } \\
\text { ( } 12 \% \text { strong recommendation for } 49 \% \text { weak } \\
\text { recommendation for) }\end{array}$ & $\begin{array}{l}\text { Moderate recommendation for } \\
\text { consensus reached when merged } \\
\text { ( } 32 \% \text { strong recommendation for } 45 \% \\
\text { weak recommendation for) }\end{array}$ \\
\hline $\begin{array}{l}\text { 11. Should patients after COVID- } 19 \text { who present with } \\
\text { interstitial abnormalities after exclusion of an active infection } \\
\text { receive an empiric systemic steroid trial? }\end{array}$ & $\begin{array}{l}\text { Weak suggestion for } \\
\text { no consensus } \\
\text { ( } 16 \% \text { strong recommendation for } 40 \% \text { weak } \\
\text { recommendation for) }\end{array}$ & $\begin{array}{l}\text { Moderate recommendation for } \\
\text { consensus reached when merged } \\
\text { ( } 14 \% \text { strong recommendation for } 59 \% \\
\text { weak recommendation for) }\end{array}$ \\
\hline $\begin{array}{l}\text { 12. Should patients after COVID- } 19 \text { who present with signs of } \\
\text { pulmonary fibrosis receive specific antifibrotic drugs? }\end{array}$ & $\begin{array}{l}\text { Suggestion for no recommendation } \\
\text { no consensus } \\
\text { (40\% no recommendation) }\end{array}$ & $\begin{array}{l}\text { Suggestion for no recommendation } \\
\text { no consensus } \\
\text { (55\% no recommendation) }\end{array}$ \\
\hline $\begin{array}{l}\text { 13. Should patients after COVID- } 19 \text { who present with persistent } \\
\text { respiratory symptoms undergo a rehabilitation program? }\end{array}$ & $\begin{array}{l}\text { Strong recommendation for } \\
\text { consensus reached }(86 \%)\end{array}$ & $\begin{array}{l}\text { Strong recommendation for } \\
\text { consensus reached }(91 \%)\end{array}$ \\
\hline
\end{tabular}




\section{Committee Discussion}

The committee discussed the need for a pulmonary follow-up in patients after hospitalization. There was agreement within the committee that a pulmonary follow-up in patients after hospitalization with COVID-19 is useful and should be performed within 3 months after initial symptoms.

A post-COVID-19 pulmonary follow-up includes, but is not limited to, specific assessment of respiratory complaints and history of COVID-19, physical examination and appropriate lung function exploration. The pulmonary follow-up can be backed by specific and generic quality of life questionnaires, specific laboratory investigations (e.g., SARS-CoV2 antibody confirmation), and imaging studies.

\section{SGP/SSP Recommendation}

- All patients hospitalized for COVID-19 should have a pulmonary follow-up. (strong recommendation for, consensus reached)

- The suggested time frame is within 3 months after infection. (suggestion, no consensus)

Remarks for Question 1: Should all patients hospitalized for COVID-19 have a pulmonary follow-up? 70\% (second survey: 77\%) strong recommendation for, 26\% (second survey: 18\%) weak recommendation for, $0 \%$ (second survey: $0 \%$ ) strong recommendation against, 5\% (second survey: 5\%) weak recommendation against, $0 \%$ (second survey: $0 \%$ ) no recommendation.

Remarks for additional question: At what time point should respiratory tests be performed? 20\% (second survey: $10 \%$ ) after 1 month, $17 \%$ (second survey: $14 \%$ ) after 2 months, 49\% (second survey: 62\%) after 3 months, $2 \%$ (second survey: $0 \%$ ) after 4 months, $12 \%$ (second survey: $14 \%)$ gave no recommendation.

\section{Question 2: Should All COVID-19 Affected Patients}

Including Those with Mild Disease Have a Pulmonary Follow-Up if Symptomatic?

\section{Literature Review}

A recent study showed that in patients who did not require hospitalization for COVID-19 pneumonia, about half remained symptomatic after 2 months and had lung functional abnormalities [28]. As most other studies and current data focus on hospitalized patients, it is difficult to evaluate lung functional impairment in patients with mild disease courses. Nevertheless, symptomatic patients might benefit from exclusion of treatable causes of dyspnea.

Swiss Recommendations for Pulmonary Long COVID
Committee Discussion

The committee discussed the necessity to further investigate patients with persisting respiratory symptoms. These investigations should take place within 3 months after symptoms of acute COVID-19.

\section{SGP/SSP Recommendation}

- All COVID-19 affected patients, including those with mild disease, should have a pulmonary follow-up if symptomatic. (strong recommendation for, consensus reached)

- The suggested time frame is within 3 months after infection. (suggestion, no consensus)

Remarks for Question 2: Should all COVID-19 affected patients including those with mild disease have a pulmonary follow-up if symptomatic? $74 \%$ (second survey: $82 \%$ ) strong recommendation for, $23 \%$ (second survey: $14 \%$ ) weak recommendation for, $2 \%$ (second survey: $0 \%$ ) strong recommendation against, $0 \%$ (second survey: $0 \%$ ) weak recommendation against, $0 \%$ (second survey: $5 \%$ ) no recommendation.

Remarks for additional question: At what time point should respiratory tests be performed? 24\% (second survey: $5 \%$ ) after 1 month, $12 \%$ (second survey: $10 \%$ ) after 2 months, $48 \%$ (second survey: $62 \%$ ) after 3 months, $5 \%$ (second survey: $0 \%$ ) after 4 months, $12 \%$ (second survey: $24 \%)$ gave no recommendation.

\section{Question 3: Should All Patients with Persistent}

Respiratory Symptoms after COVID-19 Have a

Pulmonary Follow-Up Including Plethysmography,

Diffusion Capacity Measurement, and Blood Gases

Analysis?

Literature Review

Since the beginning of the SARS-CoV2 pandemic, several cohort studies examining pulmonary function tests (PFTs) at various time points have been conducted. Included patients were mostly admitted with severe or moderate disease. The vast majority was on oxygen therapy, noninvasive ventilation, high flow nasal cannula or mechanical ventilation during the acute phase of the COVID-19. PFTs were assessed at discharge, 30 days, 4 months [29], and up to 6 months [25]. Overall, the carbon monoxide transfer capacity (TLCO) was uniformly reduced while forced vital capacity (FVC) and forced expiratory volume in $1 \mathrm{~s}$ (FEV1) were within normal range in most cases. Patients with more severe acute infection had lower TLCO value. FVC and total lung capacity (TLC) were reduced only in the patients with the most severe acute infection. Huang et al. [25] observed that $56 \%$ of the 
patients treated with mechanical ventilation had TLCO below $80 \%$ predicted after 6 months. Among those who received oxygen therapy or no oxygen, reduced TLCO was measured in 29 and $22 \%$, respectively [25]. Obstructive patterns on spirometry were not observed in these studies.

What lessons can be learned from previous coronavirus epidemics? SARS-CoV-1 and Middle East respiratory syndrome (MERS) caused a long-term decline in lung function. Between 6 months and 2 years after infection with SARS-CoV-1, there was a decrease in TLCO and TLC (restrictive syndrome) in 15.5-43.6\% and 5.2-10.9\% of cases, respectively $[3,4]$. In survivors of MERS, TLCO was still reduced in 37\% 12 months after acute infection [30].

Low TLCO in patients with LC is associated with interstitial infiltrates on chest $\mathrm{CT}$ scan. The available studies do not detail arterial blood gas analysis after acute COVID-19.

\section{Committee Discussion}

Evaluation of persistent respiratory symptoms is an indication to perform PFTs [31]. The panel specified that plethysmography should be performed on top of spirometry and diffusion capacity measurements if these are abnormal, to provide assessment of a possible restrictive ventilatory defect. The panel suggests performing arterial blood gas measurements in patients with low transcutaneous oxygen saturation at rest $\left(\mathrm{SpO}_{2}<92 \%\right)$, desaturation on exercise $\left(\mathrm{SpO}_{2}<90 \%\right)$, or receiving supplemental domiciliary oxygen therapy.

\section{SGP/SSP Recommendation}

- All patients with persistent respiratory symptoms after COVID-19 should have a pulmonary follow-up including plethysmography, diffusion capacity measurement and blood gases analysis. (strong recommendation for, consensus reached)

Remarks for Question 3: Should all patients with persistent respiratory symptoms after COVID-19 have a pulmonary follow-up including plethysmography, diffusion capacity measurement and blood gases analysis? 91\% (second survey: 100\%) strong recommendation for, 5\% (second survey: 0\%) weak recommendation for, $0 \%$ (second survey: $0 \%)$ strong recommendation against, $2 \%$ (second survey: $0 \%$ ) weak recommendation against, $2 \%$ (second survey: $0 \%$ ) no recommendation.
Question 4: Should All Patients with Severe COVID-19 (WHO Definition) Have a Pulmonary Follow-Up Including Plethysmography and Diffusion Capacity, Blood Gases Analysis Independently of Symptom Persistence?

Literature Review

Early observations found impaired diffusion capacity in patients after COVID-19 hospital discharge [29], and half of patients had impaired lung function after 30 days [32]. Our recent study showed impaired oxygen uptake up to 4 months after severe or critical COVID-19 [20]. An overview about current studies with lung functional measurements after severe disease is provided in online supplementary Table 1 (for all online suppl. material, see www.karger.com/doi/10.1159/000517255). Whether the reduced diffusion capacity is due to alveolar surface loss, interstitial fibrosis, or vascular impairment cannot be judged from this value alone [33-35]. Plethysmography is helpful to detect a restrictive ventilator defect, as observed in pulmonary fibrosis and described after COVID-19 [36]. Arterial blood gas analysis facilitates diagnosis of hypoxemia, which may be associated with impaired diffusion capacity.

Most data on lung functional impairment emerge from prospective research cohort COVID-19 follow-up studies. Recent publications indicate that pulmonary impairment is more prevalent after severe disease [20,25].

\section{Committee Discussion}

Assessment of disease impact is an additional indication to perform PFTs [31]. The committee agreed that a routine pulmonary follow-up is justified in those patients in which severe pulmonary sequelae are expected and that require evaluation for treatment.

\section{SGP/SSP Recommendation}

- For patients with severe COVID-19 (WHO definition), a pulmonary follow-up is recommended, including plethysmography and diffusion capacity, blood gases analysis independently of symptom persistence. (moderate recommendation for, consensus reached when strong and weak recommendations are merged)

Remarks for Question 4: Should all patients with severe COVID-19 (WHO definition) have a pulmonary followup including plethysmography and diffusion capacity, blood gases analysis independently of symptom persistence? $49 \%$ (second survey: 64\%) strong recommendation for, $44 \%$ (second survey: $36 \%$ ) weak recommendation for, $2 \%$ (second survey: $0 \%$ ) strong recommendation 
against, 2\% (second survey: $0 \%$ ) weak recommendation against, $2 \%$ (second survey: $0 \%$ ) no recommendation.

Question 5: Should Exercise Testing (e.g., 6MWD or Equivalent) Be Routinely Performed after COVID-19? Literature Review

Increasing evidence suggests that a range of respiratory symptoms persists in a substantial number of $\mathrm{CO}$ VID-19 survivors. Several studies suggest that these occur independently of the initial degree of severity of the disease $[23,37,38]$. Respiratory symptoms such as dyspnea occurred in up to $40 \%$ of individuals 2-6 months following COVID-19 [10, 25]. Exercise testing is commonly performed to investigate respiratory impairment, and the straightforward 6 min walking test (6MWT) is a useful tool to evaluate a range of chronic respiratory conditions [39]. Although the 6MWT has not been validated in COVID-19-associated pulmonary impairment, it is being increasingly employed to evaluate individuals after COVID-19 to diagnose exercise-related hypoxia and evaluate decreased exercise capacity $[23,25,40]$. In a Norwegian single-center study, half of the patients had hypoxia $\left(\mathrm{SpO}_{2}\right.$ $<90 \%$ ) during the 6MWT before hospital discharge [41]. Another study that evaluated patients with severe disease after 6 weeks found that walking distances in the $6 \mathrm{MWT}$ were under the predicted value for $79 \%$ of the participants, albeit without desaturation [42]. Data from a Swiss observational study acquired 4 months following severe/ critical COVID-19 also showed a decreased 6MWT distance, but with significant desaturation in such patients [20]. A Chinese study performed in 1,733 COVID-19 patients 6 months following COVID-19 observed that between $24-29 \%$ of patients had a $6 \mathrm{MWT}$ distance less than the lower normal range depending on depending on the severity of the SARS-CoV-2 infection [25]. In addition, the 6MWT has been employed as a useful investigation to monitor COVID-19 patients during rehabilitation [43, 44]. To date no guideline exists that recommends the $6 \mathrm{MWT}$ as a routine examination in patients following COVID-19.

\section{Committee Discussion}

The committee discussed that the form of exercise testing might depend on the local possibilities. A 6MWD should be performed as a minimum to detect exercise hypoxia in patients with otherwise normal lung functional testing. In case of suspected impaired oxygen uptake, CPET may be useful to determine the underlying pathology.

Swiss Recommendations for Pulmonary Long COVID
SGP/SSP Recommendation

- Exercise testing (e.g., 6MWD or equivalent) is recommended after COVID-19. (weak recommendation for, consensus reached)

Remarks for Question 5: Should exercise testing (e.g., $6 \mathrm{MWD}$ or equivalent) be routinely performed after COVID-19? 26\% (second survey: 0\%) strong recommendation for, $52 \%$ (second survey: $77 \%$ ) weak recommendation for, $7 \%$ (second survey: 0\%) strong recommendation against, 5\% (second survey: $9 \%$ ) weak recommendation against, $12 \%$ (second survey: $14 \%$ ) no recommendation.

Question 6: Should Chest CT Scan Be Routinely

Performed in Patients with Persisting Respiratory

Symptoms after COVID-19?

Literature Review

A range of radiological abnormalities have been reported following COVID-19 infection [45]. In addition to interstitial opacities, consolidations and pneumatoceles, air trapping suggestive of small airways disease and/or microvascular thrombosis has been described [45]. A 3 months' follow-up study from The Netherlands reported a reduction (but persistence) of ground-glass opacification (GGO), bronchi(ol)ectasis, lines or bands, and fibrotic changes in $86,60,64$, and $26 \%$ of participants, respectively [46]. The presence of an increased number of such abnormalities was associated with lower diffusion capacity [46]. Specifically, radiological signs of fibrosis were associated with older age, decreased TLC, and oxygen desaturation in the 6MWT, but not dyspnea [46]. In a 4 months' follow-up, we found an association between mosaic hypo-attenuation and severe/critical COVID-19 that may indicate small airways involvement and/or microvascular sequelae [20]. Although consolidations, interstitial involvement with sub-pleural lines, and ground-glass opacities had mostly resolved by 5 months, a Chinese Study on 1,733 participants observed that extensive radiological abnormalities in CT scans were associated with more severe clinical courses of COVID-19 [25]. In addition, the authors reported an increased risk of diffusion impairment, depression, fatigue, and muscle weakness in the presence of more pronounced radiological abnormalities [25]. A 6 months' follow-up reported residual CT abnormalities in $62 \%$ of patients and fibrotic-like changes in one-third of patients with severe COVID-19 [47]. Such radiographic abnormalities were associated with older age, ARDS, extensive initial chest CT scan changes, and impaired diffusion capacity [47]. Importantly, $63 \%$ of patients with ARDS developed fibrotic-type changes after 6 months, compared to $5 \%$ in non-ARDS patients [47]. 
While all the above follow-up studies provide valuable information on chest CT scan abnormalities, important questions on the long-term clinical and histologic consequences remain unanswered to date: First, do fibrotic chest CT scan abnormalities reliably identify irreversible alterations after COVID-19-related ARDS? Second, what is the significance and outcome of other, nonfibrotic chest CT scan patterns such as GGOs that have been associated with irreversible ILD [48]. Finally, how do chest CT scan patterns in post-COVID-19 ARDS and ventilator-induced lung damage differ from those abnormalities related to inflammatory pathways caused by COVID-19 that may eventually progress to lung fibrosis in a small proportion of patients?

In summary, despite an increasing number of published long-term follow-up studies, the evidence for radiological patterns that enable prediction of a clinical course and/or prognosis after COVID-19 is still insufficient.

\section{Committee Discussion}

The committee agreed that, until further data is available, follow-up chest CT imaging should be performed in patients with unexplained respiratory symptoms and functional impairment after COVID-19. The protocol of the chest CT scan needs to be defined and is subject of current evaluations.

\section{SGP/SSP Recommendation}

- Chest CT scan is recommended to be routinely performed in patients with persisting respiratory symptoms after COVID-19. (moderate recommendation for, consensus reached when strong and weak recommendations are merged)

Remarks for Question 6: Should chest CT scan be routinely performed in patients with persisting respiratory symptoms after COVID-19? 40\% (second survey: 68\%) strong recommendation for, $42 \%$ (second survey: $32 \%$ ) weak recommendation for, $2 \%$ (second survey: $0 \%$ ) strong recommendation against, $7 \%$ (second survey: $0 \%$ ) weak recommendation against, $9 \%$ (second survey: $0 \%$ ) no recommendation.

\section{Question 7: Should CPET Be Routinely Performed} after COVID-19 with Persistent Symptoms?

Literature Review

To date few observational studies addressed this question [11]. When addressing this question in July 2020, the ATS/ERS international task force provided no recommendation for or against routine cardio-pulmonary exer- cise testing, within 30-60 days post-COVID-19 pneumonia [19]. Despite an agreement that CPET would be useful for the follow-up of the patients, concerns were raised regarding the potential risks of exposing health-care workers to aerosols in case of undiagnosed COVID-19 infection. This concern does not apply to LC patients who are not carriers of an active infection. It has to be stressed that the task force was concerned exclusively about routine testing, and it recommended a case-by-case evaluation for symptomatic patients (either new or persistent symptoms). Using a $1 \mathrm{~min}$ sit-to-stand test, the Short Physical Performance Battery (to assess lower extremities function) and the Barthel index (to assess performance for activities of daily life), Belli et al. [49] reported in a small retrospective study, abnormal physical and functioning performances in $30-50 \%$ of 103 COVID-19 patients who survived hospitalization, at discharge, highlighting the need to establish an objective reference baseline, e.g. by CPET, from which to monitor recovery over time. In a letter to the editor, Gao et al. [50] reported the CPET results performed in 10 rehabilitated patients with COVID-19, one month post-discharge, having observed reduced peak $\mathrm{VO}_{2}$ in all cases and decreased oxygen pulse in a vast majority. More recently, a small retrospective German study reported CPET results obtained by testing 10 patients with persistent post-COVID-19 dyspnea. They found a reduced mean peak oxygen uptake at $73 \%$ of predicted value, a preserved mean peak work rate (92.4\% of predicted value) and a high mean value of lactate, supporting the hypothesis that post-COVID-19 dyspnea may be mostly related to muscular limitation [51]. In conclusion, there are currently no RCTs or prospective studies with sufficient numbers of patients to draw sound conclusions regarding the question of the indication to perform a routine CPET for patients with persistent symptoms after COVID-19.

\section{Committee Discussion}

The feasibility to perform routine CPET is limited in several centers. If available, the committee stresses the usefulness of CPET if unexplained gas exchange impairment is present. CPET is also helpful to determine work inability in patients with reduced physical exercise capacity. It has to be stated that the second survey was very close to reaching a consensus for a weak recommendation for performing routine $\mathrm{CPET}$.

\section{SGP/SSP Recommendation}

- CPET is recommended to be routinely performed after COVID-19 with persistent symptoms. (moderate rec- 
ommendation for, consensus reached when strong and weak recommendations are merged)

Remarks for Question 7: Should CPET be routinely performed after COVID-19 with persistent symptoms? $23 \%$ (second survey: 14\%) strong recommendation for, $42 \%$ (second survey: $68 \%$ ) weak recommendation for, $5 \%$ (second survey: 9\%) strong recommendation against, $16 \%$ (second survey: $9 \%$ ) weak recommendation against, 14\% (second survey: 0\%) no recommendation.

Question 8: Should Patients with Persistent Symptoms after COVID-19 Have Access to Specialized

Multidisciplinary Post-COVID-19 Clinics or

Networks?

Literature Review

Following the acute phase of COVID-19, approximately 1 out of 10 persons do not recover and may seek healthcare advice with a variety of symptoms such as a reduced exercise capacity, cough, thoracic pain, fatigue, and neuropsychological problems. Symptoms may overlap, affect any organ system and impact on quality of life. In the majority of patients, these symptoms resolve and rarely persist beyond 12 weeks after the acute phase. A recent US study found that only $65 \%$ of people had returned to their previous level of health 14-21 days after a positive test [52]. However, symptoms may persist for significantly longer, with fever, fatigue and breathlessness that may even recur in some individuals $[11,25,53]$. The LC syndrome refers to individuals with persistent symptoms extending beyond 4-12 weeks after the acute phase that are not explained by an alternative diagnosis $[11,15]$. These patients may need therapeutic advice with specialist investigation, rehabilitation, psychological support and further follow-up. To address these issues, post-COVID/LC assessment clinics have been established to support GPs after initial evaluation. An increasing number of LC clinics are being installed and specific guidelines have been formulated [15].

These clinics should ideally provide a multidisciplinary assessment by providing necessary resources for the care of patients recovering from COVID-19 [54]. Since the exact nature of the multiple deficits is unclear, one objective of such clinics is to ensure continuity of care and accurately track important health related outcomes. The multi-professional team comprises pulmonologists, rheumatologists, neurologists, psychiatrists, cardiologists, otolaryngologists, dermatologists, internal medicine physicians, nutritionists, physiotherapists, nephrologists, and others.

Swiss Recommendations for Pulmonary Long COVID

\section{Committee Discussion}

In general, the majority of the panel recognizes the importance of such clinics and several comments to the question highlight the importance of a multi-/interdisciplinary and multi-professional approach due to the wide range of symptoms. However, access may be limited for some patients where no such clinics exist in the vicinity. Since the benefit of such centers is not based on a body of evidence, tertiary centers should provide reasonable efforts to include patients in registries and clinical trials.

\section{SGP/SSP Recommendation}

- Patients with persistent symptoms after COVID-19 are recommended to have access to specialized multidisciplinary post-COVID-19 clinics or networks. (moderate recommendation for, consensus reached when strong and weak recommendations are merged) Remarks for Question 8: Should patients with persistent symptoms after COVID-19 have access to specialized multidisciplinary post-COVID-19 clinics or networks? $65 \%$ (second survey: $55 \%$ ) strong recommendation for, $28 \%$ (second survey: $41 \%$ ) weak recommendation for, $0 \%$ (second survey: $0 \%$ ) strong recommendation against, $2 \%$ (second survey: $0 \%$ ) weak recommendation against, $5 \%$ (second survey: $5 \%$ ) no recommendation.

Question 9: Should Patients after COVID-19 Who

Present with New Onset Obstructive Lung Disease

Have Empiric Topic Inhaled or Systemic Steroid

Treatment?

Literature Review

Bronchiolitis and bronchial inflammation are common findings with many viral diseases, specifically in children [55]. Bronchiolitis leads to discrepancies between lung opacities and poor oxygenation while compliance is preserved [56]. On chest CT, small airways disease manifests by opacities with mosaic patterns $[20,45]$. The long-term outcome of these changes and the usefulness of corticosteroids remain unclear. Prior inhaled corticosteroid (ICS) use is not significantly associated with COVID-19 incidence in patients with either COPD or asthma or early clinical outcomes among patients with COVID-19 [57-59]. However, ICS may improve COVID-19-related bronchial syndromes, like for most other viral exacerbations in asthma or COPD [60].

\section{Committee Discussion}

The frequency of patients presenting with new-onset pulmonary obstructive disease after COVID-19 is un- 
clear and not consistently addressed in the literature. Some patients might have undiagnosed underlying asthma or COPD. Nevertheless, some radiological findings suggest the presence of small airway disease. An empirical treatment trial may be performed on a case-by-case basis.

\section{SGP/SSP Recommendation}

- Patients after COVID-19 who present with new obstructive lung disease are recommended to be offered empiric topic inhaled or systemic steroid treatment. (moderate recommendation for, consensus reached when strong and weak recommendations are merged) Remarks for Question 9: Should patients after COVID-19 who present with new onset obstructive lung disease have empiric topic inhaled or systemic steroid treatment? 33\% (second survey: 36\%) strong recommendation for, $37 \%$ (second survey: $36 \%$ ) weak recommendation for, $5 \%$ (second survey: $5 \%$ ) strong recommendation against, 5\% (second survey: $9 \%$ ) weak recommendation against, $21 \%$ (second survey: $14 \%$ ) no recommendation.

Question 10: Should Patients after COVID-19 Who

Present with Persistent Cough Have Empiric Inhaled

Topic Steroids?

Literature Review

Cough is among the most common symptoms associated with viral respiratory infections. $36-43 \%$ of people with a viral upper respiratory tract infection still have cough 3 months after the infection [61]. Cough is not the primary complaint occurring after COVID-19 but has been reported. In an Italian study, 60 days after initial symptom onset, only $16 \%$ of patients complained about persistent cough [10]. Similarly, in a study of formerly hospitalized UK patients, $12 \%$ of patients reported cough 90 days after onset of initial symptoms [62]. However, in such individuals, cough is a common complaint leading to GP consultations, increased health costs, and an impaired quality of life. ICS reduce the numbers of inflammatory cells in the airways and downregulate multiple inflammatory genes encoding for cytokines, chemokines, inflammatory enzymes, and receptors [63]. Although the suppression of mucosal inflammation is associated with a clinically relevant reduction of bronchial hyperreactivity, the effect of inhaled steroids for other causes of cough remains controversial [64]. Moreover, recent guidelines differ in their recommendations of ICS for chronic cough management $[65,66]$.
The role of ICS during the acute phase of COVID-19 is different. While preparing the current guidelines (between survey 1 and survey 2), an open-label RCT investigating inhaled budesonide given within 7 days of symptom onset was pre-published [67]. The likelihood of requiring urgent care, emergency department consultation and hospitalization was significantly reduced in adults inhaling budesonide, providing first evidence for an affordable and effective intervention. Additional studies are required to confirm these findings.

\section{Committee Discussion}

Methacholine testing to investigate chronic cough in patients after COVID-19 infection is rarely performed due to increased infectious risks in case of persistent viral replication. Other causes of chronic cough should be evaluated and treated if identified. The committee discussed an empiric therapy of ICS in cough following COVID-19 and recognizes that the evidence for inhaled steroids in non-COVID-19 post-viral cough is still low, with inconsistent recommendations formulated by the pulmonary societies. No clinical trials exist in persistent cough related to COVID-19. Since ICS are easy to apply with an acceptable safety profile, a limited trial of 4-6 weeks may be an option to treat cough after COVID-19. The change between survey 1 (strong recommendation for: $12 \%$ ) and 2 (strong recommendation for: $32 \%$ ) may have been caused by the recent pre-publication about ICS in acute COVID-19 infection [67] or personal experience. In patients with preexistent asthma or eosinophilic airway disease, ICS should be continued as the mainstay of therapy.

\section{SGP/SSP Recommendation}

- Patients after COVID-19 who present with persistent cough are recommended to have empiric inhaled topic steroids. (moderate recommendation for, consensus reached when strong and weak recommendations are merged)

Remarks for Question 10: Should patients after COVID-19 who present with persistent cough have empiric inhaled topic steroids? $12 \%$ (second survey: $32 \%$ ) strong recommendation for, $49 \%$ (second survey: $45 \%$ ) weak recommendation for, $5 \%$ (second survey: $5 \%$ ) strong recommendation against, 12\% (second survey: 9\%) weak recommendation against, $23 \%$ (second survey: $9 \%$ ) no recommendation. 
Question 11: Should Patients after COVID-19 Who

Present with Interstitial Abnormalities after Exclusion

of an Active Infection Receive an Empiric Systemic

Steroid Trial?

Literature Review

Post-infectious inflammatory processes often include organizing pneumonia (OP) that may occur after $\mathrm{CO}$ VID-19 infection [68-71]. OP generally improves with oral corticosteroids and recent observations suggest that patients with interstitial abnormalities after COVID-19 may benefit from systemic steroid trials [72]. This study showed that patients with symptomatic infiltrates at 6 weeks and significant persistent functional deficits improved with an average of $26 \mathrm{mg}$ /day of prednisone with a dose-reduction scheme over 6 weeks. Such treatment was associated with a mean relative increase in transfer factor by $31.6 \%$ and FVC by $9.6 \%$, as well as significant symptomatic and radiological improvement. This observational study was not randomized but showed a marked clinical improvement of these patients in less than 12 weeks overall, after a careful initial evaluation. Furthermore, this study raises the opportunity to treat OP related lesions after COVID-19 infections with low doses systemic steroids, potentially preventing persistent symptomatic interstitial lung disease [72].

\section{Committee Discussion}

The committee recognizes a steroid trial as a possible treatment approach if, e.g., OP or bronchiolitis can be diagnosed and acute infection has been ruled out. As no RCT is currently published, this remains a case-by-case decision, which should be considered especially if lung function is impaired and the patient remains symptomatic.

SGP/SSP Recommendation

- Patients after COVID-19 who present with interstitial abnormalities after exclusion of an active infection are recommended to be evaluated to receive an empiric systemic steroid trial. (moderate recommendation for, consensus reached when strong and weak recommendations are merged)

Remarks for Question 11: Should patients after COVID-19 who present with interstitial abnormalities after exclusion of an active infection receive an empiric systemic steroid trial? $16 \%$ (second survey: 14\%) strong recommendation for, $40 \%$ (second survey: 59\%) weak recommendation for, $5 \%$ (second survey: $9 \%$ ) strong recommendation against, 26\% (second survey: 5\%) weak recommendation against, $14 \%$ (second survey: $14 \%$ ) no recommendation.

Swiss Recommendations for Pulmonary Long COVID
Question 12: Should Patients after COVID-19 Who

Present with Signs of Pulmonary Fibrosis Receive

Specific Antifibrotic Drugs?

Literature Review

Recent evidence showed that antifibrotic drugs are well tolerated and provide a benefit in in progressive fibrosing lung disease independently of the underlying pathology $[73,74]$. Pulmonary fibrosis is a complication that occurs after COVID-19 [75]. Mechanisms of disease may overlap between both type of disorders, including susceptibility in men and increased age, as well as cellular mechanisms (e.g., ACE2 involvement) [76]. This complication of COVID-19 has raised concern within the pulmonary community, although the precise frequency of complicating fibrotic lung disease is unknown with currently only case series that exist. Recent publications suggest that antifibrotic drugs may be beneficial in patients with fibrosis after COVID-19 [37, 76, 77]. Although RCTs are still lacking, several studies with nintedanib (NCT04619680, NCT04541680, NCT04338802) and pirfenidone (NCT04607928, NCT04653831) are currently ongoing.

\section{Committee Discussion}

The committee agreed that evidence from ongoing RCT should be awaited to evaluate effects of antifibrotic drugs in COVID-19-induced fibrosis. According to the committees' personal experience, fibrosis after COVID-19 is usually nonprogressive. Antifibrotic treatment may be indicated if underlying progressive ILD (i.e., IPF) has been fortuitously discovered. Otherwise, patients should be evaluated on a case-by-case basis for compassionate use.

\section{SGP/SSP Recommendation}

- It is unclear whether patients after COVID-19 who present with signs of pulmonary fibrosis should receive specific antifibrotic drugs. (suggestion for no recommendation, no consensus)

Remarks for Question 12: Should patients after COVID-19 who present with signs of pulmonary fibrosis receive specific antifibrotic drugs? 9\% (second survey: $5 \%$ ) strong recommendation for, $28 \%$ (second survey: $32 \%$ ) weak recommendation for, $7 \%$ (second survey: $0 \%$ ) strong recommendation against, $16 \%$ (second survey: $9 \%$ ) weak recommendation against, $40 \%$ (second survey: $55 \%)$ no recommendation. 
Question 13: Should Patients after COVID-19

Who Present with Persistent Respiratory Symptoms

Undergo a Rehabilitation Program?

Literature Review

Current evidence for rehabilitation after COVID-19 is weak but steadily increasing [78]. Clinical trials are ongoing to evaluate the effects of pulmonary rehabilitation after COVID-19 [11]. Interim guidance from the European Respiratory Society has already recommended post-COVID-19 rehabilitation [19]. Similarly, the German S3 Recommendations propose post-COVID-19 rehabilitation [27].

\section{Committee Discussion}

The committee strongly supports the panel decision to recommend pulmonary rehabilitation in patients with ongoing respiratory symptoms.

\section{SSP Recommendation}

- Patients after COVID-19 who present with persistent respiratory symptoms are recommended to undergo a rehabilitation program. (strong recommendation for, consensus reached)

Remarks for Question 13: Should patients after COVID-19 who present with persistent respiratory symptoms undergo a rehabilitation program? 86\% (second survey: 91\%) strong recommendation for, $7 \%$ (second survey: $5 \%$ ) weak recommendation for, $0 \%$ (second survey: $0 \%$ ) strong recommendation against, $0 \%$ (second survey: $5 \%$ ) weak recommendation against, $7 \%$ (second survey: $0 \%$ ) no recommendation.

\section{Future Directions and Research Questions}

Despite the lack of a clear definition of LC, persisting respiratory symptoms and pulmonary function impairment more than 1 month after COVID-19 may indicate LC syndrome. A broadly supported, pragmatic, and clear definition is required to standardize research and support future therapeutic strategies. Moreover, evidence for the management and treatment of pulmonary manifestations of LC is still lacking. The European Respiratory Society provided initial interim guidance on rehabilitation [19], but many aspects of disease management and specific treatment approaches remain unclear to date for practitioners. Clinical trials to address several of the questions raised by the committee are currently ongoing [11]. While awaiting additional evidence, we herein provide interim guidance for patient care based on the experience of pulmonologists from Switzerland and the results of our surveybased consensus.

\section{Conclusions}

Physicians are facing patients with symptoms and functional impairment that need to be addressed. These consensus-based recommendations provide an interim support for practitioners awaiting results from ongoing and future trials. The recommendations need to be carefully reconsidered and revised as new knowledge emerges about pulmonary LC.

\section{Acknowledgement}

Swiss COVID Lung Study Group, on behalf of SGP/SSP.

\section{Survey Participants}

Dan Adler, Andrea Azzola, Otto Brändli, Martin Brutsche, Gregory Clark, Christian Clarenbach, Patrick Fachinger, Gregoire Gex, Peter Grendelmeier, Ivan Guerreiro, Rene Hage, Raphael Heinzer, Markus Hofer, Katrin Hostettler, Jean-Paul Janssens, Werner Karrer, Rebekka Kleiner, Philipp Latzin, Irene Laube, Marco Laures, Romain Lazor, Daniele Marino, Geneviève Nicolet, Adam Ogna, Nikolay Pavlov, Alexander Pavlovic, Jerome Plojoux, Susanne Pohle, Maura Prella, Jochen Rüdiger, Otto Schoch, Dieter Scholtze, Macé Schuurmans, Thomas Sigrist, Alina Stadler, Claudia Steurer, Robert Thurnheer, Alexander Turk.

\section{Statement of Ethics}

This work does not involve human subjects and informed consent was not required. No ethical approval was necessary for this survey.

\section{Conflict of Interest Statement}

M.F.C. has received unrestricted grants and/or speaker fees from Boehringer Ingelheim, Roche and Novartis unrelated to this work. C.F.C. has received payments for lectures/speaking, development of educational materials, and travel/accommodation/ meeting expenses/ advisory boards from Roche, Novartis, Boehringer, GSK, Astra Zeneca, Sanofi, Vifor, OM Pharma, and Mundipharma. C.v.G. has received honoraria for consulting, advisory role, or lectures from AstraZeneca, Boehringer Ingelheim, GSK, Mundipharma, Novartis, OM Pharma, Pfizer, PneumRx, and Pulmonx Sanofi. The other authors have no conflicts of interest to declare in relation to this work. 


\section{Funding Sources}

No funding was received for this work.

\section{Author Contributions}

M.F.C., P.O.B, C.F.C., P.M.S., L.N., and C.v.G. drafted the questions for the Delphi survey during a virtual meeting before sending them to the participants, M.F.C. collected and evaluated the survey results. M.F.C., P.O.B., C.F.C., P.M.S., L.N., and C.v.G. drafted the first version of the manuscript, discussed the results during a virtual meeting, finalized the manuscript, and approved the last version.

\section{References}

1 Tsui PT, Kwok ML, Yuen H, Lai ST. Severe acute respiratory syndrome: clinical outcome and prognostic correlates. Emerg Infect Dis. 2003 Sep;9(9):1064-9.

2 de Wit E, van Doremalen N, Falzarano D, Munster VJ. SARS and MERS: recent insights into emerging coronaviruses. Nat Rev Microbiol. 2016 Aug;14(8):523-34.

3 Hui DS, Wong KT, Ko FW, Tam LS, Chan DP, Woo J, et al. The 1-year impact of severe acute respiratory syndrome on pulmonary function, exercise capacity, and quality of life in a cohort of survivors. Chest. 2005 Oct;128(4):2247-61.

4 Ngai JC, Ko FW, Ng SS, To KW, Tong M, Hui DS. The long-term impact of severe acute respiratory syndrome on pulmonary function, exercise capacity and health status. Respirology. 2010 Apr;15(3):543-50.

5 Huang C, Wang Y, Li X, Ren L, Zhao J, Hu Y, et al. Clinical features of patients infected with 2019 novel coronavirus in Wuhan, China. Lancet. 2020 Feb;395(10223):497-506.

6 Torres Acosta MA, Singer BD. Pathogenesis of COVID-19-induced ARDS: implications for an ageing population. Eur Respir J. 2020 Sep;56(3):2002049.

7 Herridge MS, Tansey CM, Matté A, Tomlinson G, Diaz-Granados N, Cooper A, et al.; Canadian Critical Care Trials Group. Functional disability 5 years after acute respiratory distress syndrome. $\mathrm{N}$ Engl J Med. 2011 Apr;364(14):1293-304.

8 Peter G. Gibson, Ling Qin SHP. COVID-19 acute respiratory distress syndrome (ARDS): clinical features and differences from typical pre-COVID-19 ARDS. Med J Aust. 2020 Jul;213(2):54-56.e1.

9 Berlin DA, Gulick RM, Martinez FJ. Severe Covid-19. N Engl J Med. 2020 Dec;383(25):2451-60.

10 Carfi A, Bernabei R, Landi F; Gemelli Against COVID-19. Post-Acute Care Study Group. Persistent symptoms in patients after acute COVID-19. JAMA. 2020 Aug;324(6):603-5.

11 Nalbandian A, Sehgal K, Gupta A, Madhavan MV, McGroder C, Stevens JS, et al. Post-acute COVID-19 syndrome. Nat Med. 2021 Apr;27(4):601-615.
12 Greenhalgh T, Knight M, A'Court C, Buxton M, Husain L. Management of post-acute covid-19 in primary care. BMJ. 2020 Aug;370:m3026.

13 Amenta EM, Spallone A, Rodriguez-Barradas MC, El Sahly HM, Atmar RL, Kulkarni PA. Postacute covid-19: an overview and approach to classification. Open Forum Infect Dis. 2020 Oct;7(12):a509.

14 Brodin P. Immune determinants of COVID-19 disease presentation and severity. Nat Med. 2021 Jan;27(1):28-33.

15 COVID-19 rapid guideline: managing the long-term effects of COVID-19. London: National Institute for Health and Care Excellence (UK); 2020 Dec 18.

16 Ackermann M, Verleden SE, Kuehnel M, Haverich A, Welte T, Laenger F, et al. Pulmonary Vascular Endothelialitis, Thrombosis, and Angiogenesis in Covid-19. N Engl J Med. 2020 Jul;383(2):120-8.

17 Brigham E, O’Toole J, Kim SY, Friedman M, Daly L, Kaplin A, et al. The Johns Hopkins Post-Acute COVID-19 Team (PACT): A Multidisciplinary, Collaborative, Ambulatory Framework Supporting COVID-19 Survivors. Am J Med. $2021 \mathrm{Apr} ; 134(4): 462-467 . e 1$.

18 Gorna R, MacDermott N, Rayner C, O'Hara M, Evans S, Agyen L, et al. Long COVID guidelines need to reflect lived experience. Lancet. 2021 Feb 6;397(10273):455-457.

19 Spruit MA, Holland AE, Singh SJ, Tonia T, Wilson KC, Troosters T. COVID-19: Interim Guidance on Rehabilitation in the Hospital and Post-Hospital Phase from a European Respiratory Society and American Thoracic Society-coordinated International Task Force. Eur Respir J. 2020 Aug;56(6):2002197.

20 Guler SA, Ebner L, Aubry-Beigelman C, Bridevaux PO, Brutsche M, Clarenbach C, et al. Pulmonary function and radiological features 4 months after COVID-19: first results from the national prospective observational Swiss COVID-19 lung study. Eur Respir J. 2021 Apr;57(4):2003690.

21 Wilson KC. Consensus-based recommendations in respiratory medicine. Eur Respir J. 2020;56(6):2002889.
22 Garrigues E, Janvier P, Kherabi Y, Le Bot A, Hamon A, Gouze H, et al. Post-discharge persistent symptoms and health-related quality of life after hospitalization for COVID-19. J Infect. 2020 Dec;81(6):e4-6.

23 Lerum TV, Aaløkken TM, Brønstad E, Aarli B, Ikdahl E, Aarberg Lund KM, et al. Dyspnoea, lung function and CT findings 3 months after hospital admission for COVID-19.EurRespirJ.2021 Apr29;57(4):2003448.

24 Sonnweber T, Sahanic S, Pizzini A, Luger A, Schwabl C, Sonnweber B, et al. Cardiopulmonary recovery after COVID-19 - an observational prospective multi-center trial. Eur Respir J. 2021 Apr 29;57(4):2003481.

25 Huang C, Huang L, Wang Y, Li X, Ren L, Gu $\mathrm{X}$, et al. 6-month consequences of COVID-19 in patients discharged from hospital: a cohort study. Lancet. 2021 Jan;397(10270):220-32.

26 George PM, Barratt SL, Condliffe R, Desai SR, Devaraj A, Forrest I, et al. Respiratory followup of patients with COVID-19 pneumonia. Thorax. 2020 Nov;75(11):1009-16.

27 Kluge S, Janssens U, Welte T, Weber-Carstens S, Schälte G, Spinner CD, et al. S3-Leitlinie Empfehlungen zur stationären Therapie von Patienten mit COVID-19. AWMF online. 2021

28 Trinkmann F, Müller M, Reif A, Kahn N, Kreuter M, Trudzinski F, et al.; Lung Network Rhine-Neckar-Region. Residual symptoms and lower lung function in patients recovering from SARS-CoV-2 infection. Eur Respir J. $2021 \mathrm{Feb} ; 57(2): 2003002$.

29 Mo X, Jian W, Su Z, Chen M, Peng H, Peng P, et al. Abnormal pulmonary function in $\mathrm{CO}$ VID-19 patients at time of hospital discharge. Eur Respir J. 2020 Jun;55(6):2001217.

30 Park WB, Jun KI, Kim G, Choi JP, Rhee JY, Cheon S, et al. Correlation between pneumonia severity and pulmonary complications in Middle East respiratory syndrome. J Korean Med Sci. 2018 May;33(24):e169.

31 Graham BL, Steenbruggen I, Miller MR, Barjaktarevic IZ, Cooper BG, Hall GL, et al. Standardization of Spirometry 2019 Update. An Official American Thoracic Society and European Respiratory Society Technical Statement. Am J Respir Crit Care Med. 2019 Oct;200(8):e70-88. 
32 Frija-Masson J, Debray MP, Gilbert M, Lescure FX, Travert F, Borie R, et al. Functional characteristics of patients with SARSCoV-2 pneumonia at 30 days post-infection. Eur Respir J. 2020 Aug;56(2):2001754.

33 Nusair S. Abnormal carbon monoxide diffusion capacity in COVID-19 patients at time of hospital discharge. Eur Respir J. 2020 Jul;56(1):2001832.

34 Chen R, Gao Y, Chen M, Jian W, Lei C, Zheng J, et al. Impaired pulmonary function in discharged patients with COVID-19: more work ahead. Eur Respir J. 2020 Jul;56(1):2002194.

35 Chapman DG, Badal T, King GG, Thamrin C. Caution in interpretation of abnormal carbon monoxide diffusion capacity in COVID-19 patients. Eur Respir J. 2021 Jan;57(1):2003263.

36 George PM, Wells AU, Jenkins RG. Pulmonary fibrosis and COVID-19: the potential role for antifibrotic therapy. Lancet Respir Med. 2020 Aug;8(8):807-15.

37 Townsend L, Dowds J, O’Brien K, Sheill G, Dyer AH, O'Kelly B, et al. Persistent Poor Health Post-COVID-19 Is Not Associated with Respiratory Complications or Initial Disease Severity. Ann Am Thorac Soc. 2021 Jan 8. doi: 10.1513/AnnalsATS.2020091175OC. Online ahead of print.

38 Jacobson KB, Rao M, Bonilla H, Subramanian A, Hack I, Madrigal M, et al. Patients with uncomplicated COVID-19 have long-term persistent symptoms and functional impairment similar to patients with severe COVID-19: a cautionary tale during a global pandemic. Clin Infect Dis. 2021 Feb 7;ciab103. doi: 10.1093/cid/ciab103. Online ahead of print.

39 Crapo RO, Casaburi R, Coates AL, et al.; ATS Committee on Proficiency Standards for Clinical Pulmonary Function Laboratories. ATS statement: guidelines for the six-minute walk test. Am J Respir Crit Care Med. 2002 Jul;166(1):111-7.

40 Houchen-Wolloff L, Daynes E, Watt A, Chaplin E, Gardiner N, Singh S. Which functional outcome measures can we use as a surrogate for exercise capacity during remote cardiopulmonary rehabilitation assessments? A rapid narrative review. ERJ Open Res. 2020 Dec;6(4):00526-2020.

41 Fuglebjerg NJ, Jensen TO, Hoyer N, Ryrsø CK, Lindegaard B, Harboe ZB. Silent hypoxia in patients with SARS CoV-2 infection before hospital discharge. Int J Infect Dis. 2020 Oct;99:100-1.

42 Daher A, Balfanz P, Cornelissen C, Müller A, Bergs I, Marx N, et al. Follow up of patients with severe coronavirus disease 2019 (COVID-19): pulmonary and extrapulmonary disease sequelae. Respir Med. 2020 Nov Dec;174:106197.

43 Curci C, Negrini F, Ferrillo M, Bergonzi R, Bonacci E, Camozzi DM, et al. Functional outcome after inpatient rehabilitation in post-intensive care unit COVID-19 patients: findings and clinical implications from a realpractice retrospective study. Eur J Phys Reha- bil Med. 2021 Jan 4. doi: 10.23736/S19739087.20.06660-5. Online ahead of print.

44 Curci C, Pisano F, Bonacci E, Camozzi DM, Ceravolo C, Bergonzi R, et al. Early rehabilitation in post-acute COVID-19 patients: data from an Italian COVID-19 Rehabilitation Unit and proposal of a treatment protocol. Eur J Phys Rehabil Med. 2020 Oct;56(5):633-41.

45 Ebner L, Funke-Chambour M, von Garnier C, Ferretti G, Ghaye B, Beigelman-Aubry C. Imaging in the aftermath of COVID-19: what to expect. Eur Radiol. 2021 Jun;31(6):43904392. doi: 10.1007/s00330-020-07465-6. Epub 2020 Dec 28.

46 van den Borst B, Peters JB, Brink M, Schoon Y, Bleeker-Rovers CP, Schers H, et al. Comprehensive health assessment three months after recovery from acute COVID-19. Clin Infect Dis. 2020 Nov;ciaa1750. https://doi. org/10.1093/cid/ciaa1750.

47 Han X, Fan Y, Alwalid O, Li N, Jia X, Yuan M, et al. Six-month Follow-up Chest CT Findings after Severe COVID-19 Pneumonia. Radiology. 2021 Apr;299(1):E177-86.

48 Murphy JM, Schnyder P, Verschakelen J, Leuenberger P, Flower CD. Linear opacities on HRCT in bronchiolitis obliterans organising pneumonia. Eur Radiol. 1999;9(9):1813-

49 Belli S, Balbi B, Prince I, Cattaneo D, Masocco $\mathrm{F}$, Zaccaria S, et al. Low physical functioning and impaired performance of activities of daily life in COVID-19 patients who survived hospitalisation. Eur Respir J. 2020 Oct;56(4):2002096.

50 Gao Y, Chen R, Geng Q, Mo X, Zhan C, Jian $\mathrm{W}$, et al. Cardiopulmonary exercise testing might be helpful for interpretation of impaired pulmonary function in recovered COVID-19 patients. Eur Respir J. 2021 Jan;57(1):2004265.

51 Mohr A, Dannerbeck L, Lange TJ, Pfeifer M, Blaas S, Salzberger B, et al. Cardiopulmonary exercise pattern in patients with persistent dyspnoea after recovery from COVID-19. Multidiscip Respir Med. 2021 Jan;16(1):732.

52 Tenforde MW, Kim SS, Lindsell CJ, Billig Rose E, Shapiro NI, Files DC, et al.; IVY Network Investigators; CDC COVID-19 Response Team; IVY Network Investigators. Symptom Duration and Risk Factors for Delayed Return to Usual Health Among Outpatients with COVID-19 in a Multistate Health Care Systems Network - United States, March-June 2020. MMWR Morb Mortal Wkly Rep. 2020 Jul;69(30):993-8.

53 Rovere Querini P, De Lorenzo R, Conte C, Brioni E, Lanzani C, Yacoub MR, et al. PostCOVID-19 follow-up clinic: depicting chronicity of a new disease. Acta Biomed. 2020 Jul;91 9-S:22-8.

54 National guidance for post-COVID syndrome assessment clinics. https://www.england.nhs.uk/coronavirus/wp-content/uploads/sites/52/2020/11/C0840-nationalguidance-for-post-covid-syndrome-assesment-clinics-111220.pdf
55 Tripathi S, Al-Sayyed B, Gladfelter TR. Comparative epidemiology, hospital course, and outcomes of viral respiratory infections in hospitalized pediatric patients. Indian J Med Microbiol. 2021 Jan;39(1):24-29.

56 Huang CF, Sun PT, Tay CK. Bronchiolitis and potential pathophysiological implications in coronavirus disease 2019 acute respiratory distress syndrome patients with near-normal respiratory compliance. Crit Care Med. 2020 Oct;48(10):e993-4.

57 Choi JC, Jung SY, Yoon UA, You SH, Kim MS, Baek MS, et al. Inhaled Corticosteroids and COVID-19 Risk and Mortality: A Nationwide Cohort Study. J Clin Med. 2020 Oct;9(11):E3406.

58 Avdeev S, Moiseev S, Brovko M, Yavorovskiy A, Umbetova K, Akulkina L, et al. Low prevalence of bronchial asthma and chronic obstructive lung disease among intensive care unit patients with COVID-19. Allergy. 2020 Oct; 75(10):2703-2704.

59 Li X, Xu S, Yu M, Wang K, Tao Y, Zhou Y, et al. Risk factors for severity and mortality in adult COVID-19 inpatients in Wuhan. J Allergy Clin Immunol. 2020 Jul;146(1):110-8.

60 Lipworth B, Chan R, Kuo CR. Use of inhaled corticosteroids in asthma and coronavirus disease 2019: keep calm and carry on. Ann AllergyAsthmaImmunol.2020 Nov;125(5):5034.

61 Ryan NM, Vertigan AE, Ferguson J, Wark P, Gibson PG. Clinical and physiological features of postinfectious chronic cough associated with H1N1 infection. Respir Med. 2012 Jan;106(1):138-44.

62 Arnold DT, Hamilton FW, Milne A, Morley AJ, Viner J, Attwood M, et al. Patient outcomes after hospitalisation with COVID-19 and implications for follow-up: results from a prospective UK cohort. Thorax. 2020 Dec 3;76(4):399-401.

63 Barnes PJ. How corticosteroids control inflammation: Quintiles Prize Lecture 2005. Br J Pharmacol. 2006 Jun;148(3):245-54.

64 Juniper EF, Kline PA, Vanzieleghem MA, Ramsdale EH, O'Byrne PM, Hargreave FE. Long-term effects of budesonide on airway responsiveness and clinical asthma severity in inhaled steroid-dependent asthmatics. Eur Respir J. 1990 Nov;3(10):1122-7.

65 Gibson P, Wang G, McGarvey L, Vertigan AE, Altman KW, Birring SS, et al.; CHEST Expert Cough Panel. Treatment of unexplained chronic cough chest guideline and expert panel report. Chest. 2016 Jan;149(1):27-44.

66 Morice AH, Millqvist E, Bieksiene K, Birring SS, Dicpinigaitis P, Domingo Ribas C, et al. ERS guidelines on the diagnosis and treatment of chronic cough in adults and children. Eur Respir J. 2020 Jan;55(1):1901136.

67 Ramakrishnan S, Nicolau DV, Langford B, et al. Inhaled Budesonide in the Treatment of Early COVID-19 Illness: A Randomised Controlled Trial. SSRN Electron J. 2021. doi:https://doi.org/10.2139/ssrn.3777194. 
68 Kory P, Kanne JP. SARS-CoV-2 organising pneumonia: 'Has there been a widespread failure to identify and treat this prevalent condition in COVID-19?'. BMJ Open Respir Res. 2020 Sep;7(1):e000724.

69 Vadász I, Husain-Syed F, Dorfmüller P, Roller FC, Tello K, Hecker M, et al. Severe organising pneumonia following COVID-19. Thorax. 2020 Nov 11;thoraxjnl-2020-216088. doi 10.1136/thoraxinl-2020-216088. Online ahead of print.

70 Wang Y, Jin C, Wu CC, Zhao H, Liang T, Liu $\mathrm{Z}$, et al. Organizing pneumonia of COVID-19: time-dependent evolution and outcome in CT findings. PLoS One. 2020 Nov;15(11):e0240347.

71 Horii H, Kamada K, Nakakubo S, Yamashita Y, Nakamura J, Nasuhara Y, et al. Rapidly progressive organizing pneumonia associated with COVID-19. Respir Med Case Rep. 2020;31:101295.
72 Myall KJ, Mukherjee B, Castanheira AM, Lam JL, Benedetti G, Mak SM, et al. Persistent Post-COVID-19 Interstitial Lung Disease. An Observational Study of Corticosteroid Treatment. Ann Am Thorac Soc. 2021 May;18(5):799-806.

73 Flaherty KR, Wells AU, Cottin V, Devaraj A, Walsh SL, Inoue Y, et al.; INBUILD Trial Investigators. Nintedanib in progressive fibrosing interstitial lung diseases. N Engl J Med. 2019 Oct;381(18):1718-27.

74 Maher TM, Corte TJ, Fischer A, Kreuter M, Lederer DJ, Molina-Molina M, et al. Pirfenidone in patients with unclassifiable progressive fibrosing interstitial lung disease: a double-blind, randomised, placebo-controlled, phase 2 trial. Lancet Respir Med. 2020 Feb;8(2):147-57.
75 Picchi G, Mari A, Ricciardi A, Carucci AC, Sinatti G, Cosimini B, et al. Three cases of covid-19 pneumonia in female patients in italy who had pulmonary fibrosis on follow-up lung computed tomography imaging. Am J Case Rep. 2020 Nov;21:e926921.

76 Lechowicz K, Drożdżal S, Machaj F, Rosik J, Szostak B, Zegan-Barańska M, et al. COVID-19: The Potential Treatment of Pulmonary Fibrosis Associated with SARS-CoV-2 Infection. J Clin Med. 2020 Jun;9(6):E1917.

77 Spagnolo P, Balestro E, Aliberti S, Cocconcelli E, Biondini D, Casa GD, et al. Pulmonary Fibrosis Secondary to COVID-19: A Call to Arms? Lancet Respir Med. 2020 Aug;8(8):750752.

78 Negrini F, De Sire A, Andrenelli E, Lazzarini SG, Patrini M, Ceravolo MG. Rehabilitation and COVID-19: a rapid living systematic review 2020 by Cochrane Rehabilitation Field. Update as of October 31st, 2020. Eur J Phys Rehabil Med. 2021 Feb;57(1):166-170. 